\title{
New Results from the Cosmic-Ray Program of the NA61/SHINE facility at the CERN SPS
}

\author{
Michael Unger* for the NA61/SHINE Collaboration ${ }^{\dagger}$ \\ Karlsruhe Institute of Technology (KIT), Postfach 3640, D-76021 Karlsruhe, Germany \\ E-mail: michael.ungerakit.edu
}

The NA61/SHINE experiment at the SPS accelerator at CERN is a unique facility for the study of hadronic interactions at fixed target energies. The data collected with NA61/SHINE is relevant for a broad range of topics in cosmic-ray physics including ultrahigh-energy air showers and the production of secondary nuclei and anti-particles in the Galaxy.

Here we present an update of the measurement of the momentum spectra of anti-protons produced in $\pi^{-}+\mathrm{C}$ interactions at 158 and $350 \mathrm{GeV} / c$ and discuss their relevance for the understanding of muons in air showers initiated by ultrahigh-energy cosmic rays.

Furthermore, we report the first results from a three-day pilot run aimed at investigating the capability of our experiment to measure nuclear fragmentation cross sections for the understanding of the propagation of cosmic rays in the Galaxy. We present a preliminary measurement of the production cross section of Boron in $\mathrm{C}+\mathrm{p}$ interactions at $13.5 \mathrm{AGeV} / \mathrm{c}$ and discuss prospects for future data taking to provide the comprehensive and accurate reaction database of nuclear fragmentation needed in the era of high-precision measurements of Galactic cosmic rays.

36th International Cosmic Ray Conference -ICRC2019-

July 24th - August 1st, 2019

Madison, WI, U.S.A.

\footnotetext{
${ }^{*}$ Speaker.

${ }^{\dagger}$ http://shine.web.cern.ch/content/author-list
} 


\section{Introduction}

NA61/SHINE ${ }^{1}$ [1] is a multi-purpose fixed target experiment to study hadron production in hadron-nucleus and nucleus-nucleus collisions at the CERN Super Proton Synchrotron (SPS).

The main part of the detector consists of a set of large-acceptance Time Projection Chambers (TPCs) and two superconducting magnets with a combined bending power of $9 \mathrm{Tm}$ resulting in a precise measurement of particle momenta $\left(\sigma(p) / p^{2} \approx(0.3-7) \times 10^{-4} \mathrm{GeV} / c^{-1}\right)$ and providing excellent particle identification capabilities via the specific energy loss in the TPC volumes.

The experiment started operating in 2007 and has since collected hadroproduction data with a variety of projectiles, beam energies and target materials. Two data taking campaigns were dedicated specifically to cosmic-ray research and will be discussed in more detail in the next two sections. In addition, NA61/SHINE performed a detailed measurement of particle production in $\mathrm{p}+\mathrm{C}$ interactions at $31 \mathrm{GeV} / c[2,3,4]$ and $\pi^{+}+\mathrm{C}$ at $60 \mathrm{GeV} / c[5]$ to determine the beam properties in accelerator-based neutrino experiments. Since carbon is a good proxy for interactions on nitrogen in air, the measured spectra in these reactions are also highly relevant for the modeling of low energy interaction in air showers $[6,7]$. Furthermore, $p+p$ interactions were measured in a wide range of beam momenta $(20,31,40,80$ and $158 \mathrm{GeV} / c)[8,9]$ with the aim of defining a reference data set for the heavy ion physics program of NA61/SHINE. This data set is also very useful for the study of the secondary production of anti-protons $[10,11,12]$ and anti-nuclei $[13,14]$ in collisions of cosmic-ray protons with the interstellar medium in the Galaxy.

\section{Pion Interactions in Air Showers}

A long-standing problem in the physics of ultrahigh-energy cosmic rays is the understanding of muons in air showers, which are found to be more abundant in data than in simulated air showers (see [15] for a summary of recent measurements). The majority of muons in air showers is created in decays of charged pions when the energy of the pion is low enough such that its decay length is smaller than its interaction length. At ultra-high energies it takes several generations of interactions until the average pion energy is sufficiently small to allow for pion decays. As a result, the total number of muons in an air shower depends on details of hadronic interactions along a chain of interactions. Hence even small differences in the assumed properties of hadronic interactions can lead to a sizable effect on the predicted muon number when escalated over several generations in the particle cascade (see also discussion in [16]).

The vast majority of hadronic interactions in air showers are $\pi+$ air interactions and the muons observed by particle detectors of surface detectors mostly originate from decays of pions that got produced in pion-air interactions at equivalent beam energies below a $\mathrm{TeV}[17,6,18]$. Therefore, new data with pion beams at 158 and $350 \mathrm{GeV} / c$ on a thin carbon target (as a proxy for nitrogen) were collected by the NA61/SHINE experiment and we previously reported on the production spectra of charged hadrons and identified particles $\left(\pi^{ \pm}, \mathrm{K}^{ \pm}, \mathrm{p}(\overline{\mathrm{p}}), \Lambda(\bar{\Lambda}), \mathrm{K}_{S}^{0}, \mathrm{~K}^{* 0}, \omega\right.$ and $\left.\rho^{0}\right)[19$, $20,21,22,23,24]$.

The key to model muon production in air showers is to correctly predict the fraction $f$ of the energy that remains in the hadronic cascade in each interaction and is not lost to the electromagnetic

\footnotetext{
${ }^{1}$ SHINE $=$ SPS Heavy Ion and Neutrino Experiment
} 


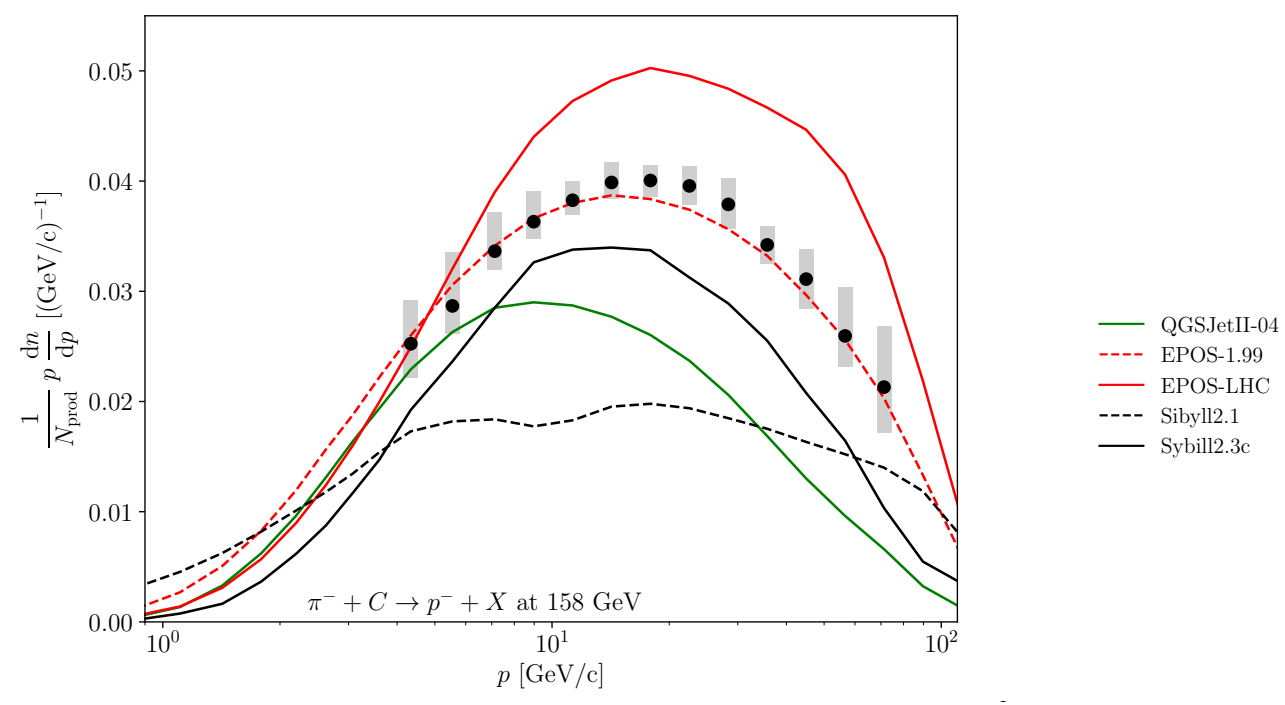

Figure 1: Comparison of the $p_{\mathrm{T}}$-integrated $\overline{\mathrm{p}}$ spectrum at $158 \mathrm{GeV} / c$ with predictions of hadronic interaction models used in air shower simulations.

component via $\pi^{0}$ production. In a simplified model with the production of only charged and neutral pions, this fraction is $f=2 / 3$ and after $n$ interactions $(2 / 3)^{n}$ of the initial energy is left in the hadronic component. Muons are produced when the pions reach low energies and decay, which happens at about $n=8$ interactions for an air shower of $10^{20} \mathrm{eV}$ [25]. In a more realistic scenario the energy transfer to the hadronic component to $f \sim(2 / 3+\Delta)$, where $\Delta$ accounts for hadronic particles without dominant electromagnetic decay channels such as $\rho^{0}$ mesons [26, 27] or baryons [28]. Then a fraction of $(2 / 3+\Delta)^{n} \approx(2 / 3)^{n}(1+3 / 2 n \Delta)$ of the initial cosmic-ray energy can produce muons after $n$ interactions and only if the value of $\Delta$ is accurately known throughout the whole chain of interactions, there is hope for a precise prediction of the muon number in air showers.

A good proxy for the baryons produced in interactions (as opposed to being struck off the target) are anti-baryons. Our previous preliminary measurements of $\bar{p}$ production in $\pi^{-}+C$ interactions had sizable systematic uncertainties due to a model-dependent correction for the feed-down of antiprotons from electroweak decays, mainly from $\bar{\Lambda} \rightarrow \bar{p}+\pi^{-}$. Here we present an update of this measurement with a data-driven correction for the feed-down. For this purpose we first measured the $\bar{\Lambda}$ production in $\pi^{-}+C$ interactions [24] and then re-weighted the model correction accordingly as described in [4]. The result is shown in Fig. 1 and compared to predictions of hadronic interaction models used in air shower simulations. As can be seen, none of the most recent versions of the models describes the data well, the older version 1.99 of EPOS, however, gives a very good description of our data. The current "LHC" version of this model overproduces anti-protons and hence the enhanced transfer to the hadronic cascade and the corresponding larger amount of muon production in this model is strongly disfavored by our data. On the other hand, all the remaining models under-produce anti-protons and thus it can be conjectured that an increase of anti-baryon production in these models to match our measurement could alleviate the muon deficit reported by air shower experiments. 

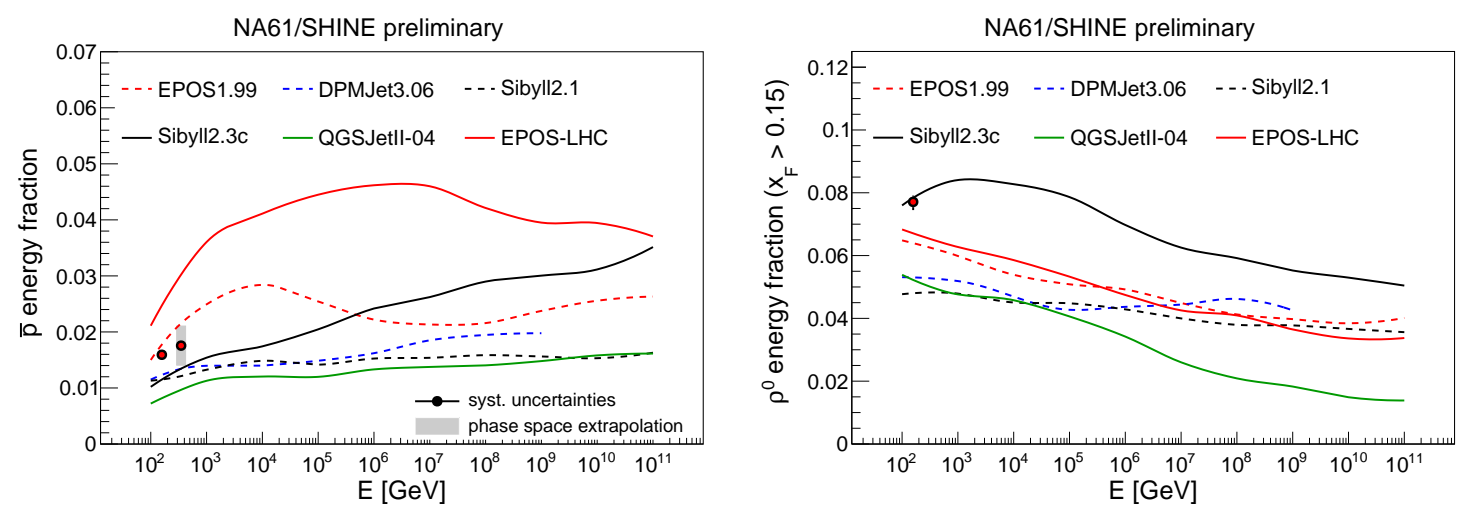

Figure 2: Energy fraction transferred to anti-protons (left) and $\rho^{0}$-mesons (right) as measured by NA61/SHINE (data points) and as predicted by hadronic interaction models over the whole range of beam energies relevant for air showers.

The total energy fractions transferred to anti-protons and $\rho^{0}$ mesons is displayed in Fig. 2. These fractions were obtained by integrating the $p \mathrm{~d} n / \mathrm{d} p$ spectra including an extrapolation up to the full beam momentum $[29,23]$. Note that the anti-proton fraction measured here constrains the production of $\mathrm{p}, \overline{\mathrm{p}}, \mathrm{n}$ and $\overline{\mathrm{n}}$ production. The sum of the energy fractions of these particles is at about the same level as the one going into $\rho^{0}$ mesons. The comparison to predictions of hadronic models reveals that none of the existing attempts to describe interactions in air showers succeeds to reproduce both energy fractions at the same time. And although the energy dependence of the energy fractions will remain a source of uncertainty for the foreseeable future, a re-tune of these models to match the low energy measurements from NA61/SHINE will significantly reduce the uncertainties in predictions of muons in air showers.

\section{Results from Pilot Run on Nuclear Fragmentation}

Recent progress in the detection of Galactic cosmic rays with space-based detectors such as PAMELA and AMS has lead to a wealth of new data on the fluxes of leptons, nuclei and antiprotons from $\mathrm{GeV}$ to $\mathrm{TeV}$ with an unprecedented percent-level precision. These new data sets provide a unique diagnostic of cosmic-ray propagation in the Galaxy and an opportunity to find signatures of astrophysical dark matter annihilation. The amount of particle production in the Galaxy depends on the integrated traversed matter density which can be inferred from the ratio of secondary to primary cosmic rays such as the ratio of Boron to Carbon if the nuclear fragmentation cross section of the primary particles are known from laboratory measurements. The current uncertainties in the modeling of the propagation of cosmic rays in the Galaxy due to uncertainties of these cross sections is however at the level of 10-20\% [30, 31, 32, 33] and thus much larger than the uncertainty of the cosmic-ray data itself.

To remedy this situation, the NA61/SHINE Collaboration is studying the possibility of performing new precise measurements of nuclear fragmentation at the CERN SPS. A pilot run [34] took place in December 2018 and we took 3 days of test data for the measurement of Boron production in $\mathrm{C}+\mathrm{p}$ interactions. 

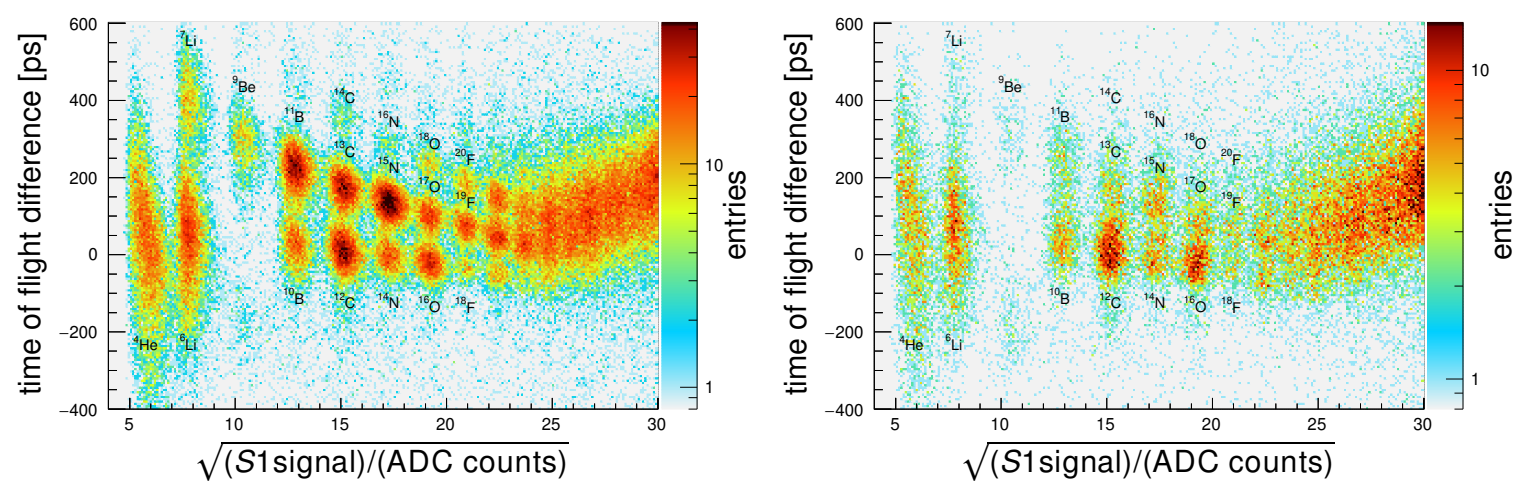

Figure 3: Beam composition recorded with free-running trigger detectors for different settings of the $\mathrm{H} 2$ beamline. Left: $E / Z=28 \mathrm{GV}, 20 \mathrm{~cm}$ primary Be target, right: $E / Z=27 \mathrm{GV}, 16 \mathrm{~cm}$ primary Be target. The signal on the $\mathrm{x}$-axis is proportional to the charge of the beam particle, whereas the $\mathrm{y}$-axis separates the isotopes masses via a measurement of the particle velocity.

For this purpose, the SPS delivered secondary ions to our experiment that were created by fragmenting a primary lead beam on a primary beryllium target (see [35] for a description of previous data taking of NA61 with a secondary ion beam). A measurement of the resulting beam composition at the experiment is shown in Fig. 3 for two different beam rigidities $R=E / Z$ and primary target thicknesses. As can be seen, even for the uncalibrated online data shown here, the beam isotopes are well separated by measuring their time of flight with two scinitillators separated by $236 \mathrm{~m}$ and the amplitude of the scintillator signals which is proportional to the squared charge of the particle. The settings on the right panel were used for the fragmentation run presented here as it maximized the number of ${ }^{12} \mathrm{C}$ ions in the beamline. The main beam trigger was set to select carbon isotopes.

More than $1.1 \times 10^{6}$ carbon beam triggers were recorded during three days of data taking alternating between three target positions as indicated on the left panel of Fig. 4: A $1 \mathrm{~cm} \mathrm{C-target,}$ a $1.5 \mathrm{~cm}$ polyethylene $\left(\mathrm{CH}_{2}\right)$ target and an empty target holder. The latter position is used to correct the measurement for out-of-target interactions and the subtraction of $\mathrm{C}+\mathrm{C}$ interactions from $\mathrm{C}+\mathrm{CH}_{2}$ interactions yields the desired fragmentation cross sections in $\mathrm{C}+\mathrm{p}$ interactions (details of the analysis can be found in [36]).

The identification of fragments downstream of the target is illustrated in the right panel of Fig. 4. Here the $\mathrm{x}$-axis shows the deviation of the measured track from the nominal beam position after the passage through the two superconducting magnets. This deviation is a measure of the rigidity of the particle relative to the beam rigidity and thus of the mass of the produced isotope. On the y-axis, the square root of the energy deposit in the TPC can be seen, which gives a measure of the charge of the particle. Since the data were taken in zero-bias mode, most tracks are in the ${ }^{12} \mathrm{C}$-peak appearing as a black area in the truncated color scale. ${ }^{11} \mathrm{C},{ }^{11} \mathrm{~B}$ and ${ }^{10} \mathrm{~B}$ fragments are clearly visible and also $\mathrm{Be}$ and $\mathrm{Li}$ fragments, though the statistics of the pilot run is too small to discern the different isotopes of the latter by eye.

As a first step, we concentrate the analysis on the sum of B isotopes. The fully calibrated $Z^{2}$ distribution derived from the energy deposit in the TPC located $10 \mathrm{~m}$ downstream of the target is shown in Fig. 5. As can be seen, the different charges are well separated with almost no overlap 

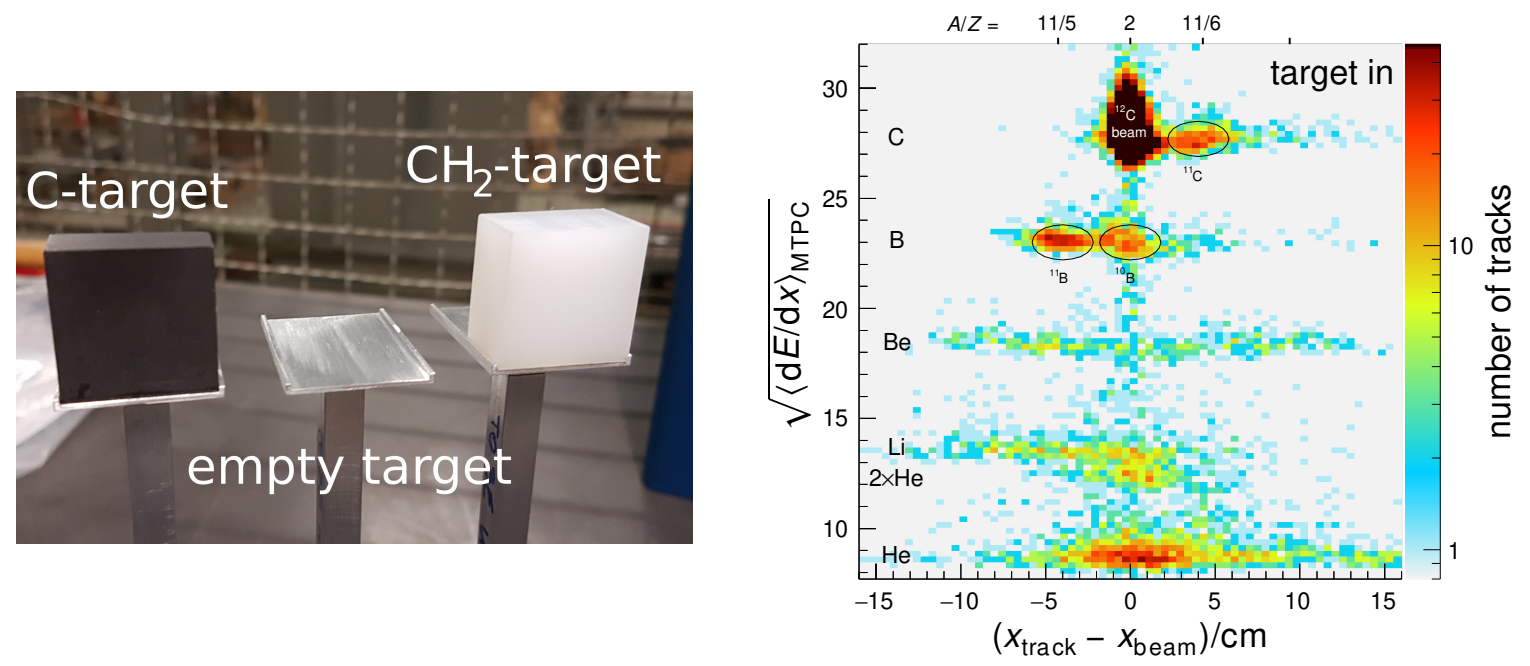

Figure 4: Left: target holder used for the data taking, right: detection of fragments in the TPC downstream of the magnetic field (see text)

between the elements. The gray histogram shows the distribution of charges without inserted target, i.e. isotopes that got produced in interactions with the detector material. The data points correspond to the charge distribution with inserted target and the red arrows indicate the range in $Z^{2}$ used to select B nuclei.

From the number of $\mathrm{B}$ nuclei produced in $\mathrm{C}+\mathrm{C}$ and $\mathrm{C}+\mathrm{CH}_{2}$ interactions we derived a preliminary measurement of the fragmentation cross section of $\mathrm{C}$ on $\mathrm{p}$ to $\mathrm{B}$ :

$$
\sigma_{\mathrm{C}+\mathrm{p} \rightarrow \mathrm{B}+X}=(47.7 \pm 3 \text { (stat.) } \pm 2.3 \text { (syst.) }) \mathrm{mb} .
$$

This measurement is compared to previous data in Fig. 6 and we find good agreement of our data with the extrapolation of the cross section derived in [33] indicated by the shaded bands. It is worthwhile noting that the asymptotic value of the cross section above $10 \mathrm{AGeV} / \mathrm{c}$ is very important to interpret the $\mathrm{B} / \mathrm{C}$ ratio measured by AMS up to $\gtrsim \mathrm{TeV}$ [37]. In this energy range only one measurement from [38] was available prior to our pilot run.

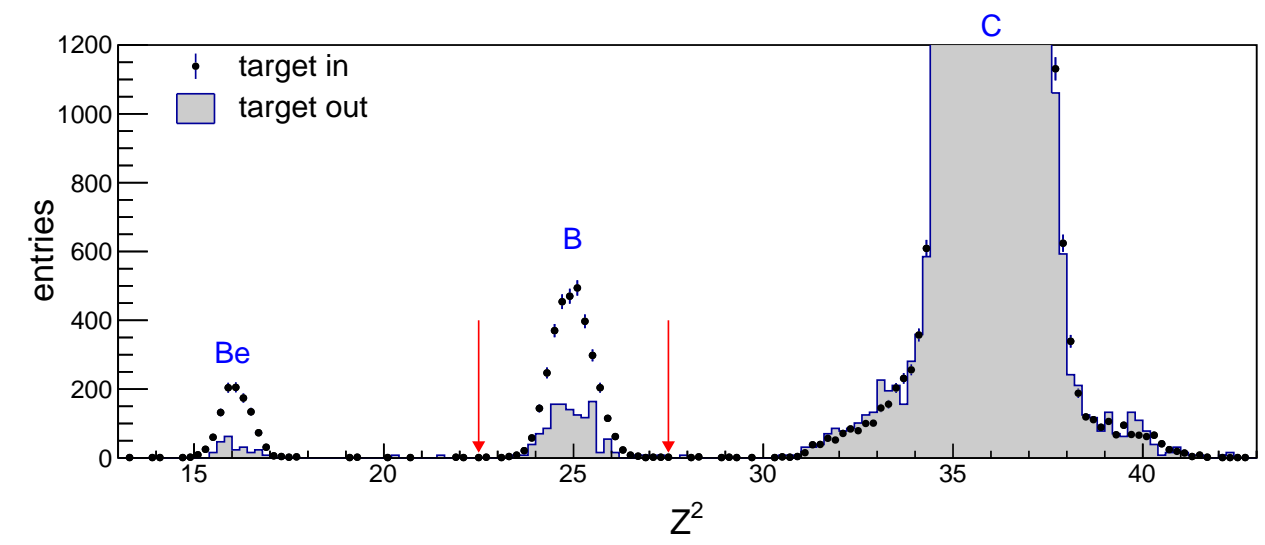

Figure 5: Distribution of the squared track charge measured with the TPC located about $10 \mathrm{~m}$ downstream of the target (see text). 


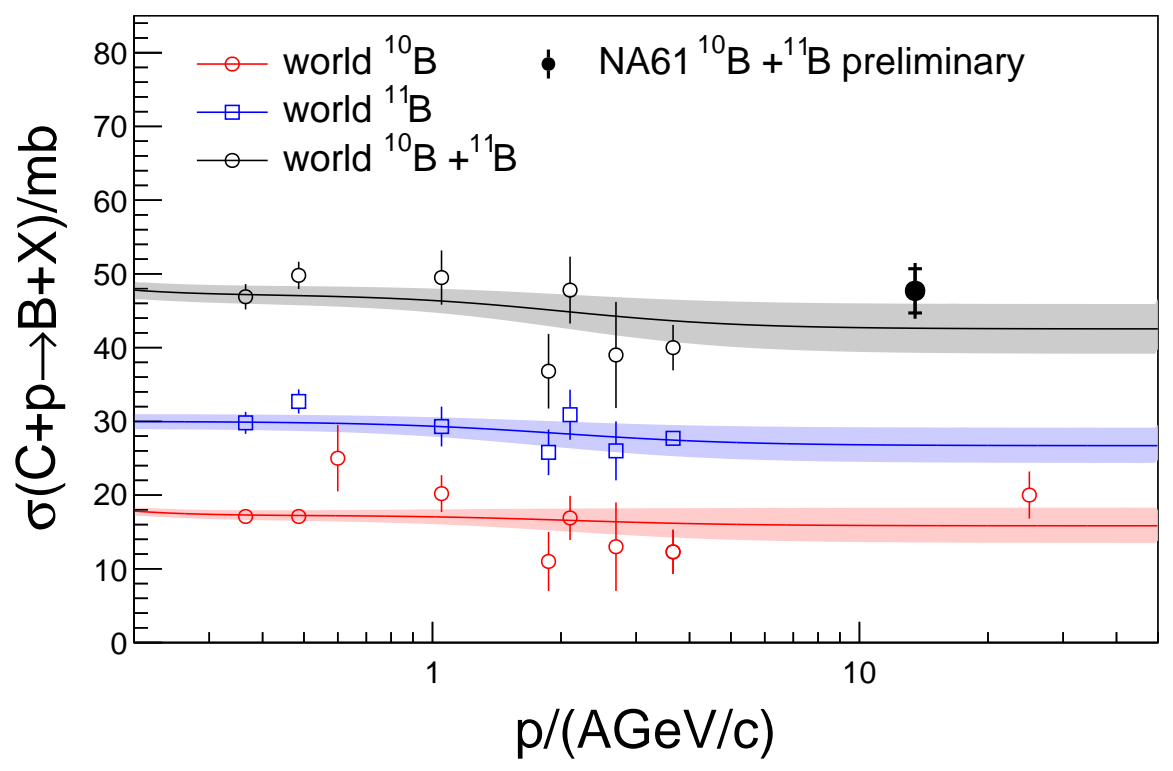

Figure 6: Measured fragmentation cross section of $\mathrm{C}+\mathrm{p} \rightarrow \mathrm{B}+X$ compared to previous measurements (open data points) and the parameterization of the fragmentation cross section from (see [33] and references therein).

\section{Summary and Outlook}

The NA61/SHINE facility at the CERN SPS provides unique opportunities to measure hadronic processes relevant for cosmic ray physics at fixed target energies. With the updated corrections to the anti-proton spectra presented here we conclude our extensive studies of the interactions of pion projectiles, which are the most numerous particles in cosmic-ray induced air showers. We presented preliminary results from a pilot study for the measurement of the fragmentation of nuclei relevant for the propagation of cosmic rays in the Galaxy. The quality of the test data demonstrates that NA61/SHINE is well suited for these measurements.

As detailed in [39], a whole range of nuclear reactions needs to be measured to allow for a precise measurement of light secondary nuclei in cosmic rays. The variety of secondary fragments delivered by the $\mathrm{H} 2$ beamline (cf. Fig. 3) is ideal to collect this data at the SPS with a single beam setting in a short time. Such a measurement campaign is proposed to take place when the LHC (and thus SPS) resume operation after the current "long shutdown" [40]. The envisaged data taking will benefit from the planned upgrade of the read-out system of NA61 to increase the possible data taking rate by a factor of ten, bringing it up to $1 \mathrm{kHz}$. At this readout speed a modest beam time of about two weeks of data taking with secondary ions from the SPS would lead to a comprehensive measurement of the most relevant cross sections for the secondary production of the cosmic-ray isotopes of $\mathrm{Li}, \mathrm{Be}, \mathrm{B}, \mathrm{C}$ and $\mathrm{N}$. 


\section{Acknowledgments}

We would like to thank the organizers for the opportunity to present these results at ICRC19 and Carmello Evoli for providing the data tables and fits shown in Fig. 6. Furthermore we would like to thank the CERN BE and EN Departments, in particular Reyes Fernandez and Nikolaos Charitonidis, for their outstanding support of NA61/SHINE without which none of the measurements presented here would have been possible.

\section{References}

[1] N. Abgrall et al., [NA61/SHINE Collab.] JINST 9 (2014) P06005.

[2] N. Abgrall et al., [NA61/SHINE Collab.] Phys. Rev. C84 (2011) 034604.

[3] N. Abgrall et al., [NA61/SHINE Collab.] Phys. Rev. C85 (2012) 035210.

[4] N. Abgrall et al., [NA61/SHINE Collab.] Eur. Phys. J. C76 (2016) 84.

[5] A. Aduszkiewicz et al., [NA61/SHINE Collab.], "Measurements of hadron production in $\pi^{+}+\mathrm{C}$ and $\pi^{+}+$Be interactions at $60 \mathrm{GeV} / \mathrm{c}, " 2019$. CERN-EP-2019-198.

[6] C. Meurer et al. Czech. J. Phys. 56 (2006) A211.

[7] M. Unger, [NA61/SHINE Collab.] PoS ICHEP2010 (2010) 449.

[8] N. Abgrall et al., [NA61/SHINE Collab.] Eur. Phys. J. C74 (2014) 2794.

[9] A. Aduszkiewicz et al., [NA61/SHINE Collab.] Eur. Phys. J. C77 (2017) 671.

[10] M. Korsmeier, F. Donato, and M. Di Mauro Phys. Rev. D97 (2018) 103019.

[11] A. Cuoco et al. Phys. Rev. D99 (2019) 103014.

[12] M. Kachelriess, I. V. Moskalenko, and S. Ostapchenko, 2019. arXiv:1904.05129.

[13] P. von Doetinchem J. Phys. Conf. Ser. 718 (2016) 042061.

[14] D.-M. Gomez-Coral et al. Phys. Rev. D98 (2018) 023012.

[15] L. Cazon, [EAS-MSU, IceCube, KASCADE- Grande, NEVOD-DECOR, Pierre Auger, SUGAR, Telescope Array and Yakutsk Collab.]. PoS ICRC2019 (2019) 214.

[16] L. Cazon. PoS ICRC2019 (2019) 005.

[17] H.-J. Drescher and G. Farrar Astropart. Phys. 19 (2003) 235.

[18] I. Maris et al. Nucl. Phys. Proc. Suppl. 196 (2009) 86.

[19] M. Unger, [NA61/SHINE Collab.] Proc 32nd ICRC 5 (2011) 67.

[20] H. Dembinski, [NA61/SHINE Collab.] Proc. 33rd ICRC (2013) 688.

[21] A. Herve, [NA61/SHINE Collab.] PoS ICRC2015 (2016) 330.

[22] A. Aduszkiewicz et al., [NA61/SHINE Collab.] Eur. Phys. J. C77 (2017) 626.

[23] R. R. Prado, [NA61/SHINE Collab.] PoS ICRC2017 (2018) 315.

[24] R. R. Prado, [NA61/SHINE Collab.] EPJ Web Conf. 208 (2019) 05006.

[25] J. Matthews Astropart. Phys. 22 (2005) 387. 
[26] H.-J. Drescher Phys.Rev. D77 (2008) 056003.

[27] S. Ostapchenko EPJ Web Conf. 52 (2013) 02001.

[28] T. Pierog and K. Werner Phys. Rev. Lett. 101 (2008) 171101.

[29] R. R. Prado. "Experimental studies of the muonic component of extensive air showers", PhD Thesis, University Sao Paulo, 2018.

[30] Y. Genolini et al. A\&A 580 (2015) A9.

[31] N. Tomassetti Phys. Rev. D96 (2017) 103005.

[32] A. Reinert and M. W. Winkler JCAP 1801 (2018) 055.

[33] C. Evoli, R. Aloisio, and P. Blasi Phys. Rev. D99 no. 10, (2019) 103023.

[34] A. Aduszkiewicz et al., [NA61/SHINE Collab.], "Feasibility Study for the Measurement of Nuclear Fragmentation Cross Sections with NA61/SHINE at the CERN SPS," 2017. CERN-SPSC-2017-035 ; SPSC-P-330-ADD-9.

[35] H. Ströbele and I. Efthymiopoulos. CERN Cour.52N4 (2012) 33.

[36] F. Sutter. "Messung der Fragmentation von Kohlenstoff auf Protonen zu Bor bei einer Energie von 13.5 A GeV", Master Thesis, Karlsruhe Institute of Technology, 2019.

[37] M. Aguilar et al., [AMS Collab.] Phys. Rev. Lett. 117 no. 23, (2016) 231102.

[38] P. Fontes Phys. Rev. C15 (1977) 2159.

[39] Y. Genolini et al. Phys. Rev. C98 (2018) 034611.

[40] A. Aduszkiewicz et al., [NA61/SHINE Collab.], “"Study of Hadron-Nucleus and Nucleus-Nucleus Collisions at the CERN SPS: Early Post-LS2 Measurements and Future Plans"," 2018.

CERN-SPSC-2018-008. SPSC-P-330-ADD-10. 